\title{
Digital twin of the process of thermal cutting of flat material into figured parts
}

\author{
M A Verkhoturov ${ }^{1}$, G N Verkhoturova ${ }^{1}$, D R Zaripov ${ }^{1}$, N V Kondratyeva ${ }^{2}$ and S S Valeev ${ }^{1,2}$ \\ ${ }^{1}$ Ufa State Aviation Technical University, K. Marx Str. 12, Ufa, 450008, Russia \\ ${ }^{2}$ Sochi State University, Politekhnicheskaya Str. 7, Sochi, 354008, Russia
}

verhotur@vmk.ugatu.ac.ru

\begin{abstract}
The designing a digital twin of the process of the thermal cutting of sheet material using laser or gas equipment for its figure cutting is considered. The solution to the problem of optimizing the path of the cutting tool taking into account the thermal effects on the material to be cut is discussed. The solution of the problem of modeling the temperature change of the material to be cut is considered on the basis of a discrete - logical representation of information about the state of the technological system of sheet cutting. The results of a computational experiment are presented.
\end{abstract}

\section{Introduction}

The process of creating any complex technical product begins with the manufacture of its elementary structural components - parts. Parts represent the bulk of production facilities for an industrial enterprise; therefore, in the entire production cycle of creating a product, the stage of their manufacture is one of the most responsible and complex, which is aggravated by the fact that a number of products are produced in a single and small-scale production.

Due to the large nomenclature of parts in production, certain difficulties arise in the organization of technological design of cutting-part operations, which include the procedure for cutting sheet material [1].

In the process of cutting sheet materials into figured parts, one of the most important stages is the construction of the path of the cutting tool. Optimization of the path of the cutting tool can reduce the cost of cutting and improve the quality of the resulting parts [2-4].

For the manufacture of billets and parts from sheet materials, figure-cutting machines with numerical control are used. The laser cutting has gained particular popularity, based on a focused laser beam, which heats the surface at a specified point to the temperature of evaporation of the material. Moving the laser beam along a given route creates a cut of the material of the desired shape $[5,6]$.

Usually, path optimization boils down to minimizing the idle stroke of the machine and reducing the points of insertion, however, this route does not take into account the observance of the temperature regime of the material.

Depending on the order of cutting out the figures, the temperature of the material to be cut can vary greatly in different local areas and cause overheating of the material with its subsequent possible deformation, which can lead to deterioration in the quality of the resulting blanks $[7,8]$. 
In this regard, it becomes necessary to take into account the thermal factor, which makes it possible to improve the quality of the obtained blanks in comparison with the usual minimization of the idle path $[9,10]$.

When solving various technological problems, various optimization methods based on digital twins are used in the framework of Industry 4.0 [11, 12]. A digital twin is a virtual model of processes, environments, objects, systems and people, synchronized with them and designed to track their past state in order to predict their future state with a given accuracy [13].

One of the directions for the development of digital twins is objects and processes of industrial production, for which it is rather difficult to determine or measure a number of parameters of their state during the execution of technological processes [14].

On the other hand, if we talk about the use of digital twins, it is necessary to "synchronize" with the technological process under consideration, in our case, the cutting process, and, first of all, with the real temperature that exists at certain points of sheet material and at a particular moment of time.

\section{The structure of an automated system for placing geometric objects}

The technological process associated with the problem of placing geometric objects at the external level can be represented as follows (see figure 1).

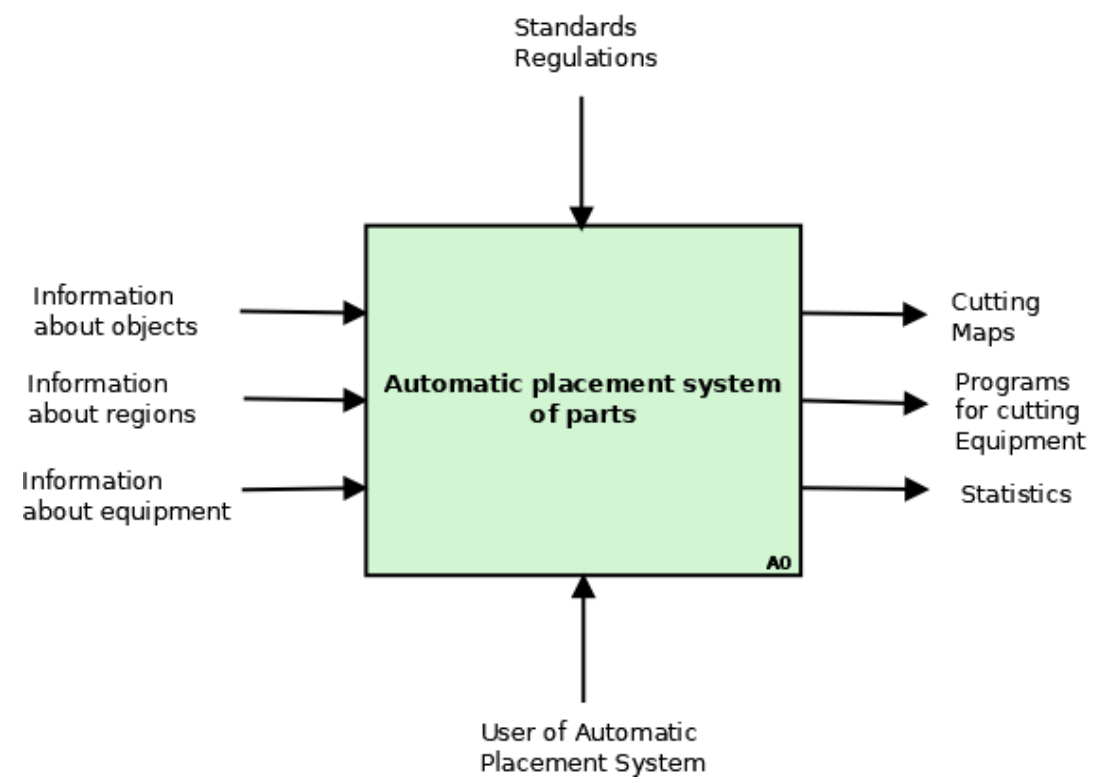

Figure 1. Representation of an automated placement system using IDEF0.

The following information is supplied to the input of the system under consideration:

- geometric parameters of cut (packed) objects;

- geometric parameters of the placement area;

- the number of objects to be placed;

- the number of areas of placement;

- type of equipment.

The control parameters are:

- technological limitations, for example, the permissible heating temperature of sheet material;

- characteristics of the consumption of sheet material.

Executors in our case can be:

- engineers of the CAD department;

- engineers of the technological department;

- engineers of the department of management of production processes.

The system output is the following information:

- geometric parameters of cutting-packing cards; 
- detailed rates of material consumption;

- coefficients of cutting and material use;

- programs for machine tools with numerical control, etc.

The internal organization can be represented in the form of two functional blocks: an optimization block and a block for processing data on technological constraints. This is due to the fact that, in addition to the optimization one, the problem is solved, connected with the need to take into account the fact that the resulting cutting-packing maps must take into account. For example, the conditions of non-intersection of the placed objects with each other and with the boundary of the region, which carry the geometric complexity of solving the problem, restrictions on the permissible temperature of the sheet during its cutting, restrictions on the movement of the cutting tool.

\section{Statement of the problem}

Let the following parameters be given:

- rectangular area to cut $L(a \times b)$;

- parts (billets) $\left\{S_{i}\right\}$, where $i=1 \div n$ is the part number;

- parts contours $\left\{C_{i j}\right\}$, where $i$ is the part number, $j$ is the number of part contours $i, j=1 \div m_{i}$ $\left(m_{i} \geq 1\right.$ is the number of outer and inner contours of the part $\left.S_{i}\right)$;

- insertion points into contours $\left\{P_{i j}\right\}$ where $i$ is the part number, $j$ is the number of part contour $S_{i}$;

- starting position of the cutting tool $P_{\text {start }}$;

- maximum acceptable temperature $t_{M A X}$.

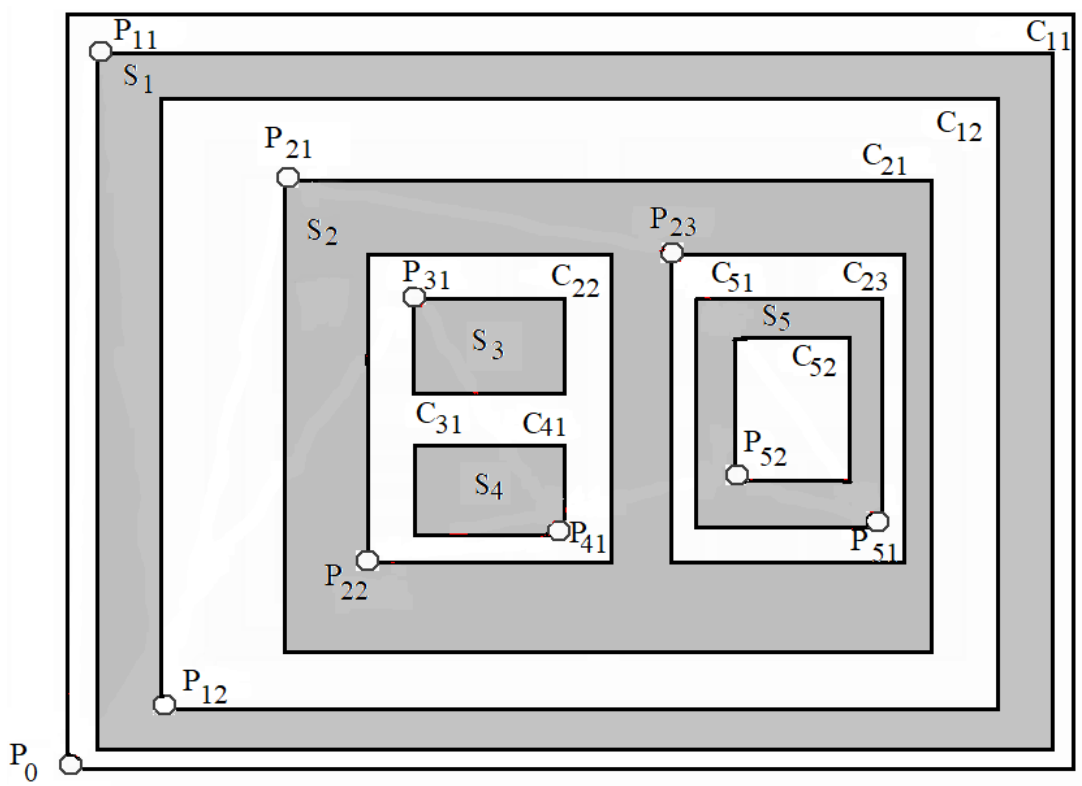

Figure 2. Parts (billets) and contours.

In figure 2 we use the following notations: $S_{1}$ is the 1st billet, $C_{11}$ and $C_{12}$ are outer and inner contours of $S_{1}$, respectively; $S_{2}$ is the 2 nd billet, $C_{21}$ is the outer contour of $S_{2}, C_{22}, C_{23}$ are inner contours of $S_{2} ; S_{3}$ is the 3rd billet, $C_{31}$ is the outer contour of $S_{3} ; S_{4}$ is the 4th billet, $C_{41}$ is outer contour of $\mathrm{S}_{4} ; \mathrm{S}_{5}$ is the 5 th billet, $\mathrm{C}_{51}$ and $\mathrm{C}_{52}$ are outer and inner contours of $\mathrm{S}_{5}$, respectively.

To find (see figure 3 ):

Such $R^{*}$ that $T\left(\operatorname{Tr}\left(R^{*}\right), L\right) \leq t_{\max } i j$ where:

- $R=\left(P_{\text {start }}, \ldots, P_{i j}, \ldots, P_{\text {start }}\right)$ is sequence of contour inset points, where $i=1 \div n, j=1 \div m_{i}$;

- $\operatorname{Tr}(R)$ is cutter path when moving along a sequence of points $R$;

- $\operatorname{T(Tr}(\cdot), L)$ is function determining the point $(x, y)$ maximum temperature $t_{\max x, y}$ sheet to be cut $L$ during cutting along the path $\operatorname{Tr}(\cdot)$. 


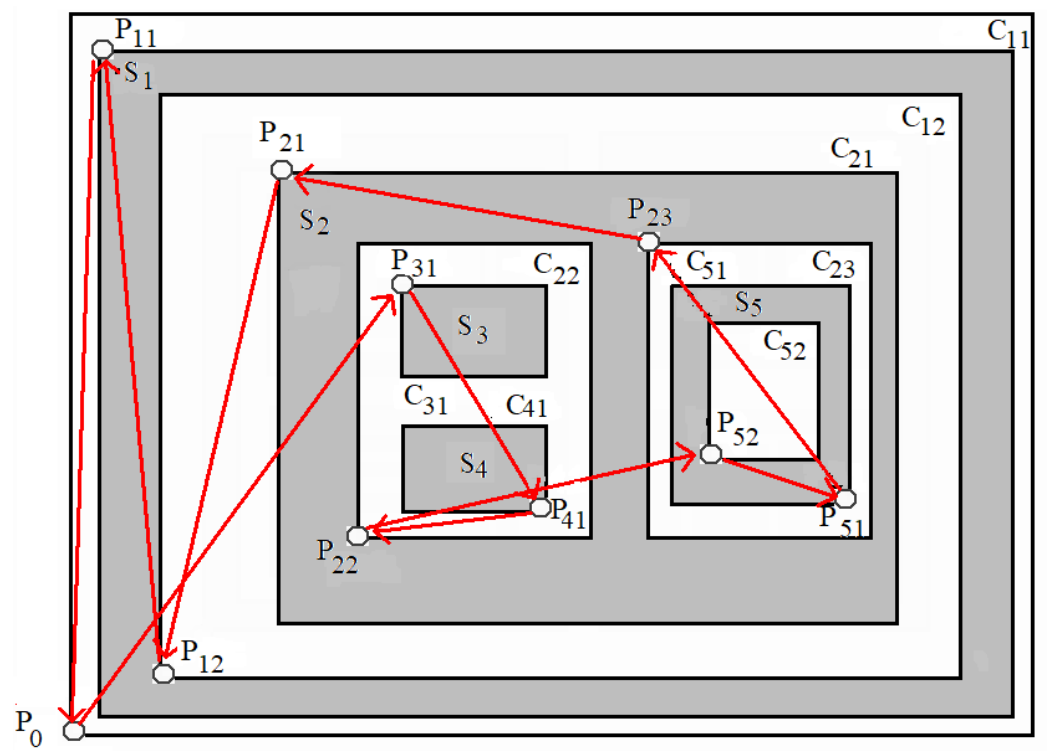

Figure 3. Cutting plan ( $S_{i}$ workpieces, $C_{i j}$ contours, $P_{i j}$ insertion points) and cutting tool path (arrows), built taking into account connectivity constraints.

It should be noted that the problem may not have a solution at certain values of $t_{\max i j}$ less than a certain value $t_{M A X}$, since when cutting, the metal heats up to the melting point and this temperature spreads over the entire area of the sheet. Accordingly, when the cutting tool moves at a given speed along all the blanks to be cut, a situation may arise when there will be no regions of the sheet with a temperature less than $t_{M A X}$-delta. If you start cutting a part (billet) located in such a zone, the temperature will rise more than the specified value $t_{\max i j}$. In practice, this problem can be solved if only to suspend cutting for a while until certain zones of the sheet have cooled to an acceptable temperature, which is usually difficult to implement in practice. It should be noted that it is rather difficult to measure the temperature at various points of the sheet. In this context, it makes sense to talk about minimizing the level of overheating, and not about "not exceeding" $t_{\max i j}$ value which is further used in this paper.

Additional restrictions on the "connectivity" of the billets/contours that are inside other contours/ billets with the "main" part of the sheet (inside the cut billets/contours there should be no uncut contours/billets) due to the following (see figure 3):

- billets/contours that are not connected to the sheet after cutting can be displaced/deformed from the temperature effect and the presence of internal stresses existing in the sheet from the moment of its manufacture, respectively, the billets/contours that are inside the cut out contours/billets, in this case, will also offset, i.e. incorrect;

- overheating of this billet/contour and the contours/parts inside it in the process of subsequent cutting of the contours/billets inside the considered ones, since there is no connection with the "main" material, which would be the heat dissipation point.

Let $Q_{i j}$ be a sequential number of the contour $C_{i j}$ cutting $\left(Q_{\downarrow i j}>0, Q_{\downarrow i j} \in Z\right)$. When going from contour $C_{i j}$ to path $C_{k l}: Q_{k l}-Q_{i j}=1$.

Constraints of the "inner-outer" connectivity: when constructing the path of the cutting tool, you must first cut out all the inner contours of the billets, and then process its outer contour:

$$
\forall C_{k j}, C_{k 1}: Q_{k 1}-Q_{k j}=1, j>1 \text {. }
$$

Herewith the following condition must be met:

$$
\forall C_{k b}: b>1 \exists Q_{k b}: Q_{k b} \leq Q_{k j}, 0<Q_{k 1}-Q_{k b}<m_{k} .
$$


Constraints of the "outer-inner" connectivity: let $I N_{C_{i l}}=\left\{D_{k}\right\}$ be the set of all billets (parts) inside the inner contour $C_{i l}$ of the billet $S_{i}$ at the first nesting level. Before you start cutting the contour $C_{i j}$, one need to cut out all the billets that are in the set $I N_{C_{i l}}$ :

$$
\forall C_{k 1}: D_{k} \in I N_{C_{i l}}, l \in\left(1 ; m_{i}\right] \exists Q_{k 1}: Q_{k 1}<\min \left\{Q_{i l}\right\}_{l=2}^{m_{i}} \text {. }
$$

\section{Solution method}

In this setting, the problem is one of the most well-known discrete optimization problems - the Traveling salesman problem (TSP) and belongs to the class of NP-hard. The main problem in solving it is the difficulty of determining the temperature of any point of the sheet in the process of cutting it along one or another chosen path. It is not possible to solve this problem analytically. In this regard, an approach based on a discrete-logical representation of information was chosen, which was tested and showed good results in solving problems of two-dimensional and three-dimensional placement of objects of complex geometric shapes [9]. In the simplest case, the matrix of points is two-dimensional, and for more accurate modeling of sheet heating and temperature propagation over it, a threedimensional matrix can be used, and the size of the third coordinate $Z$ depends on the thickness of the material.

Consider next the two-dimensional case.

Heating of the cutting area during cutting. When the cutting tool moves along the path $\operatorname{Tr}(\cdot)$, those points of the cutting area (sheet $L$ ), through which it passes during the cutting of the contour $C_{i j}$, are heated to the melting temperature of the corresponding material.

Temperature spread. The following principle of temperature spread in discrete space is proposed:

1) For each area of 9 points (with 8 -connectivity), the point $P$ with the highest temperature is selected (figure 4(a)).

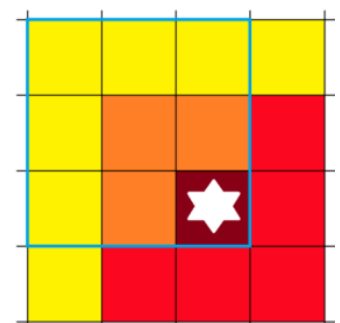

$a$

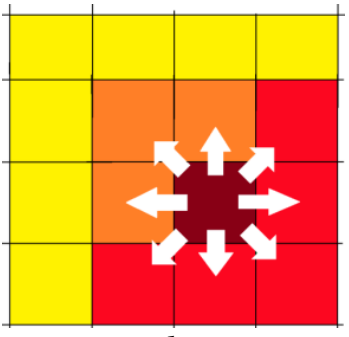

$b$

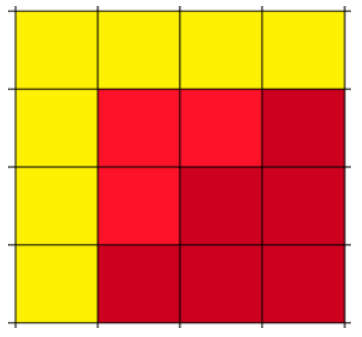

C

Figure 4. Selection of the point with the highest temperature value (a), the direction of heat propagation (b) and the temperature in the vicinity of the selected point (c).

2) Then the new temperature of the selected point $P$ is calculated depending on:

- $\quad$ point temperature $T_{P}$;

- amount of heat transferred to each neighboring point $\Delta T$;

- number of neighboring points count;

- coefficient of thermal conductivity $K_{T . c o n d}\left(0 \leq K_{\text {T.cond }} \leq 1\right)$;

- heat transfer to the environment coefficient $K_{\text {H.transf }}\left(0 \leq K_{\text {H.transf }} \leq 1\right)$;

- $\Delta T=T_{P} K_{T . c o n d}$;

- $T_{P}=\left(T_{P}-\Delta T \cdot\right.$ count $) K_{\text {H.transf. }}$

3) For each of the neighboring points $S$ (figure 4(b)), the temperature is calculated taking into account the increment $\Delta T$ depending on the thermal conductivity coefficient $K_{T \text {.cond }}$ and heat transfer to the environment coefficient $K_{\text {H.transf }}($ figure $4(\mathrm{c})): T_{S}=\left(T_{S}+\Delta T\right) K_{H . t r a n s f}$.

Synchronization with the technological process. To solve this problem, it is necessary to have information about the real temperature of the sheet material, at a particular moment in time of movement of the cutting tool, in a particular set of specified points. In the simplest case, for example, 
these can be the corner of the sheet. During the cutting process, the temperature obtained with the digital twin is corrected based on the measured temperature, and then these changes are taken into account for other points of the sheet using the above method. The current position of the cutter can also be synchronized with the actual speed and position.

Below is the pseudo-code of the algorithm and for the implementation of "Additional restrictions" (see figure 5).
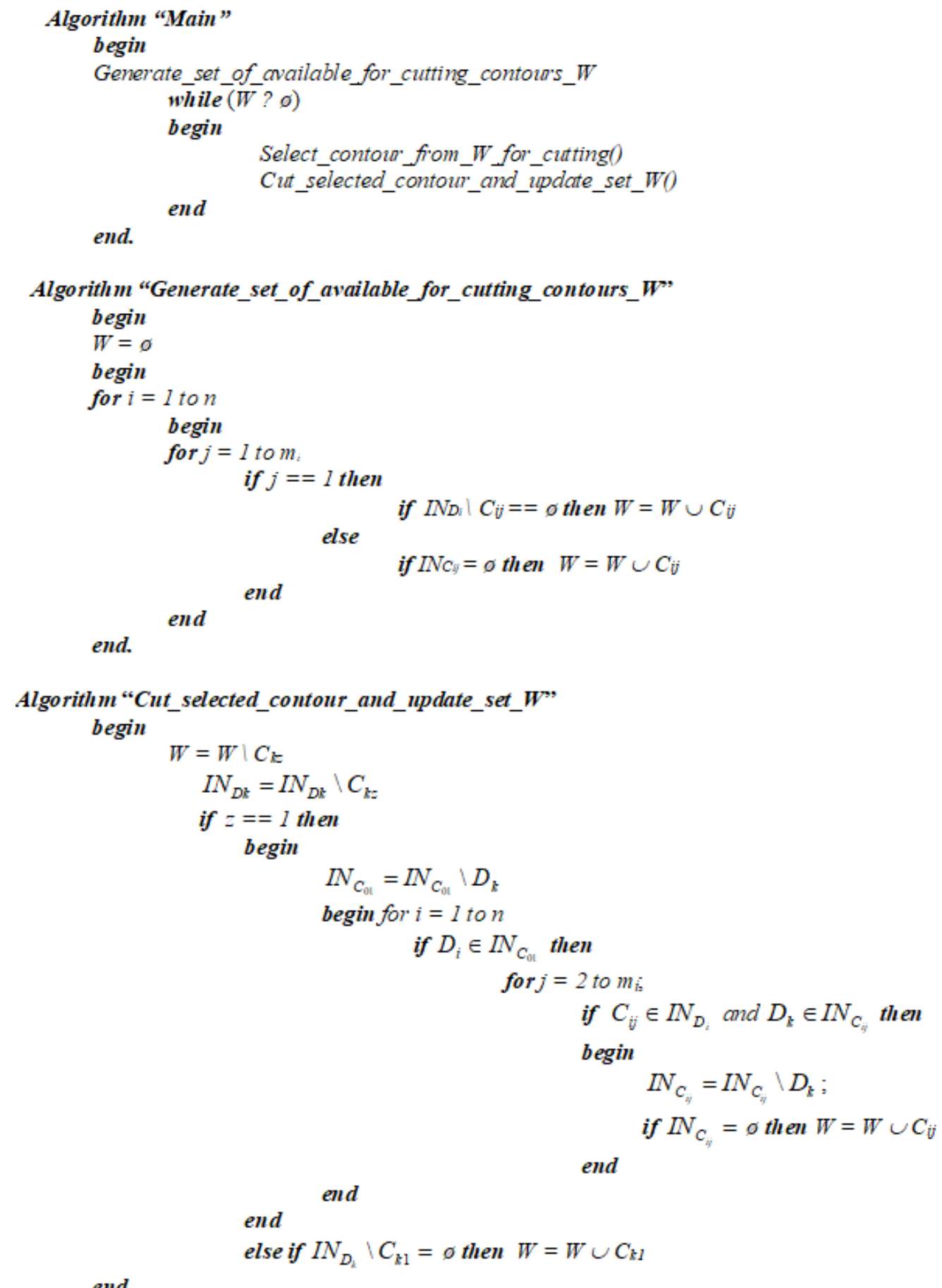

Figure 5. The pseudo-code of the algorithm and for the implementation of "Additional restrictions". 


\section{Computational experiment}

To analyze the effectiveness of the developed methods and algorithms, a computational experiment was carried out on the basis of an example from [10].

The number of local overheating of the material $G$ was chosen as an indicator of the quality of cutting taking into account thermal effects. Four algorithms were compared: "Shortest path", "Minimize overheating", "Random transitions" and "Minimize path and overheats taking into account temperature in real time" developed in this work and based on the determination of the temperature value on the scale of a numerical experiment.

Cutting plan with visualization of heating during cutting is shown in figure $6-8$.

Figure 9 shows an example of a cutting plan with the "Additional restrictions" algorithm taken into account and presented in figure 5 .

The results of computational experiments are presented in the table below. The comparison of the algorithms was carried out according to the criteria of the length of the idle path of the cutting tool $S$ and the number of local overheating $P$.

Table. The results of computational experiments

\begin{tabular}{lcccc}
\hline & $\begin{array}{c}\text { Algorithm 1: } \\
\text { Shortest path }\end{array}$ & $\begin{array}{c}\text { Algorithm 2: } \\
\text { Random } \\
\text { transitions }\end{array}$ & $\begin{array}{c}\text { Algorithm 3: } \\
\text { Minimize } \\
\text { overheating }\end{array}$ & $\begin{array}{c}\text { Algorithm 4: } \\
\text { Minimize path and } \\
\text { overheats taking into } \\
\text { account temperature } \\
\text { in real time }\end{array}$ \\
\hline $\begin{array}{l}\text { Idling path } \\
\text { length } \boldsymbol{S} \text { (cm) }\end{array}$ & 2788.184 & 33787.5 & 45952.97 & 11176.35 \\
$\begin{array}{l}\text { Number of local } \\
\text { overheating } \boldsymbol{P}\end{array}$ & 518 & 460 & 451 & 456 \\
\hline
\end{tabular}

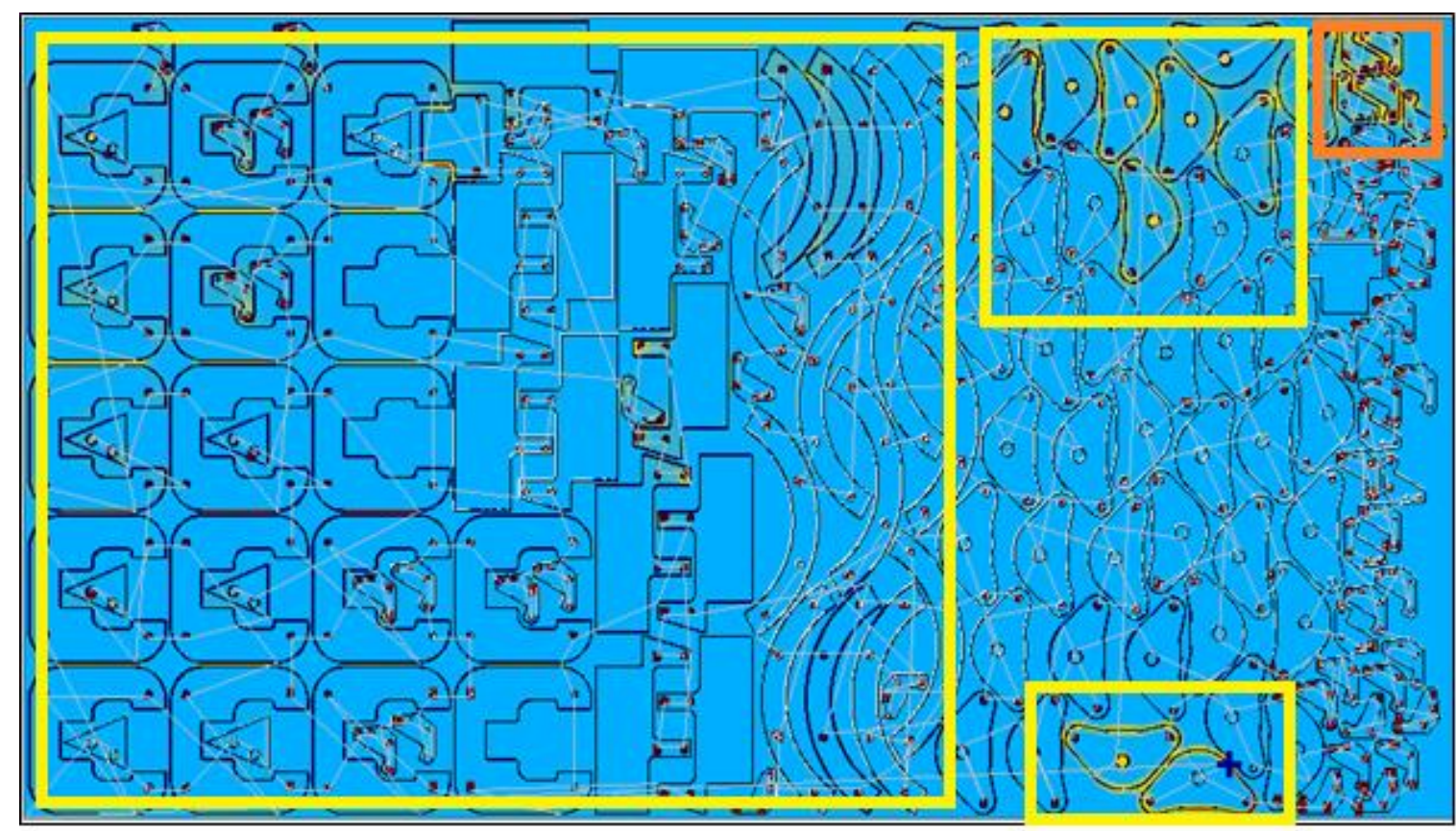

Temperature

\section{Normal Medium High}

Figure 6. Example of a cutting plan (cutter path, sheet point temperature and overheating areas). 


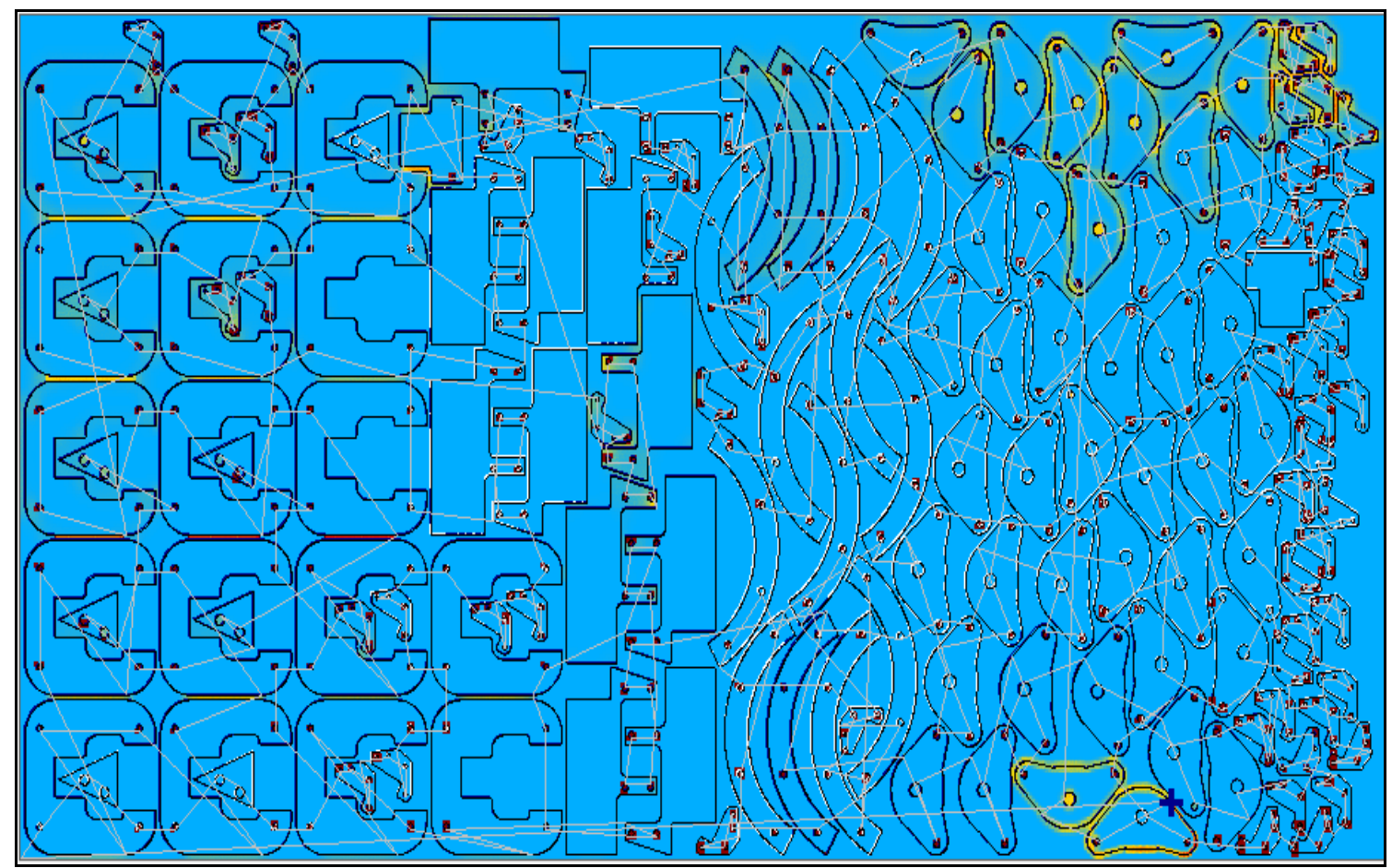

$a$

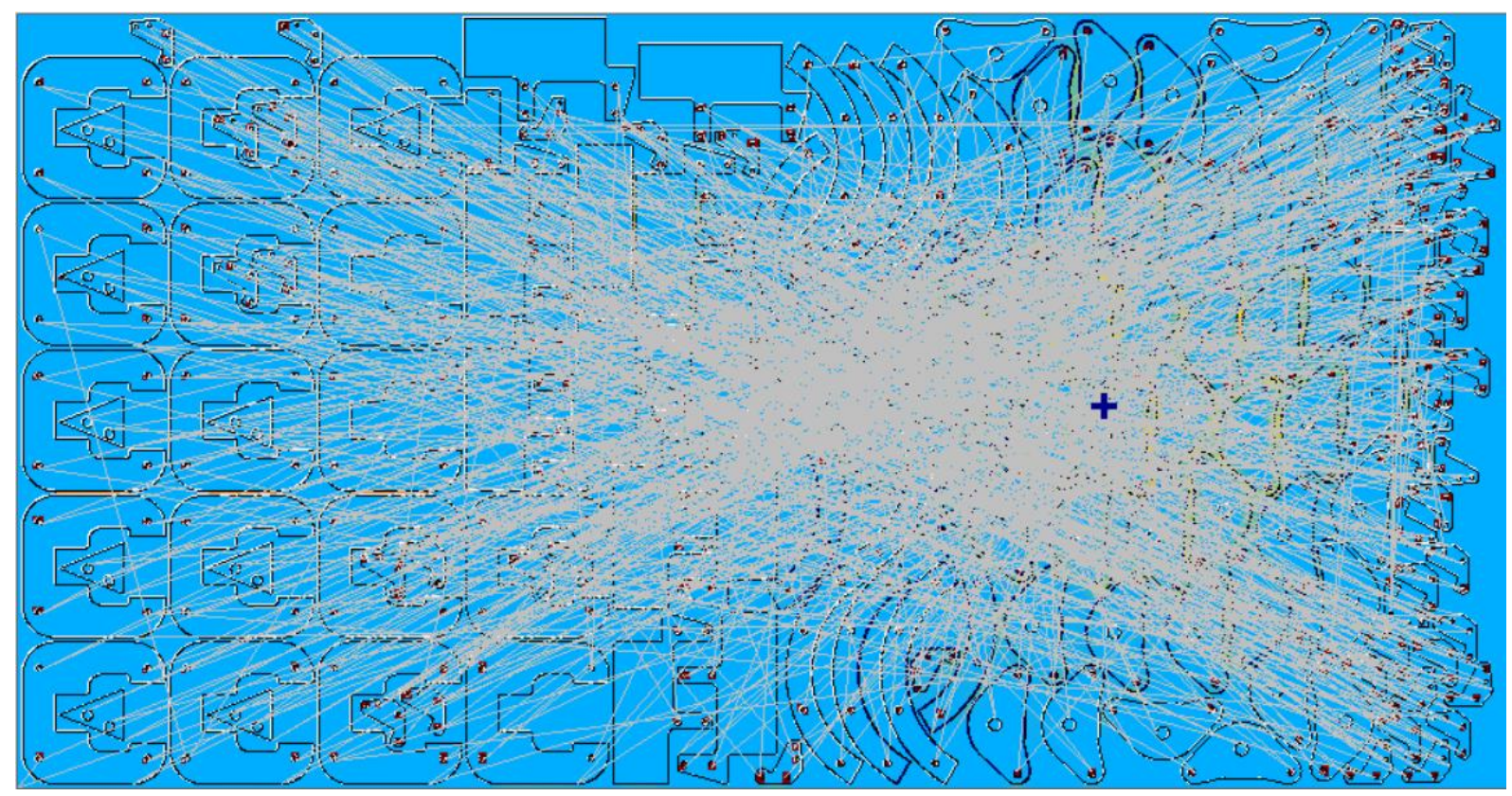

$b$

Figure 7. Examples of a cutting plan: cutting plan obtained by the "Shortest path" algorithm (a) and the "Minimize overheating" algorithm (b). 


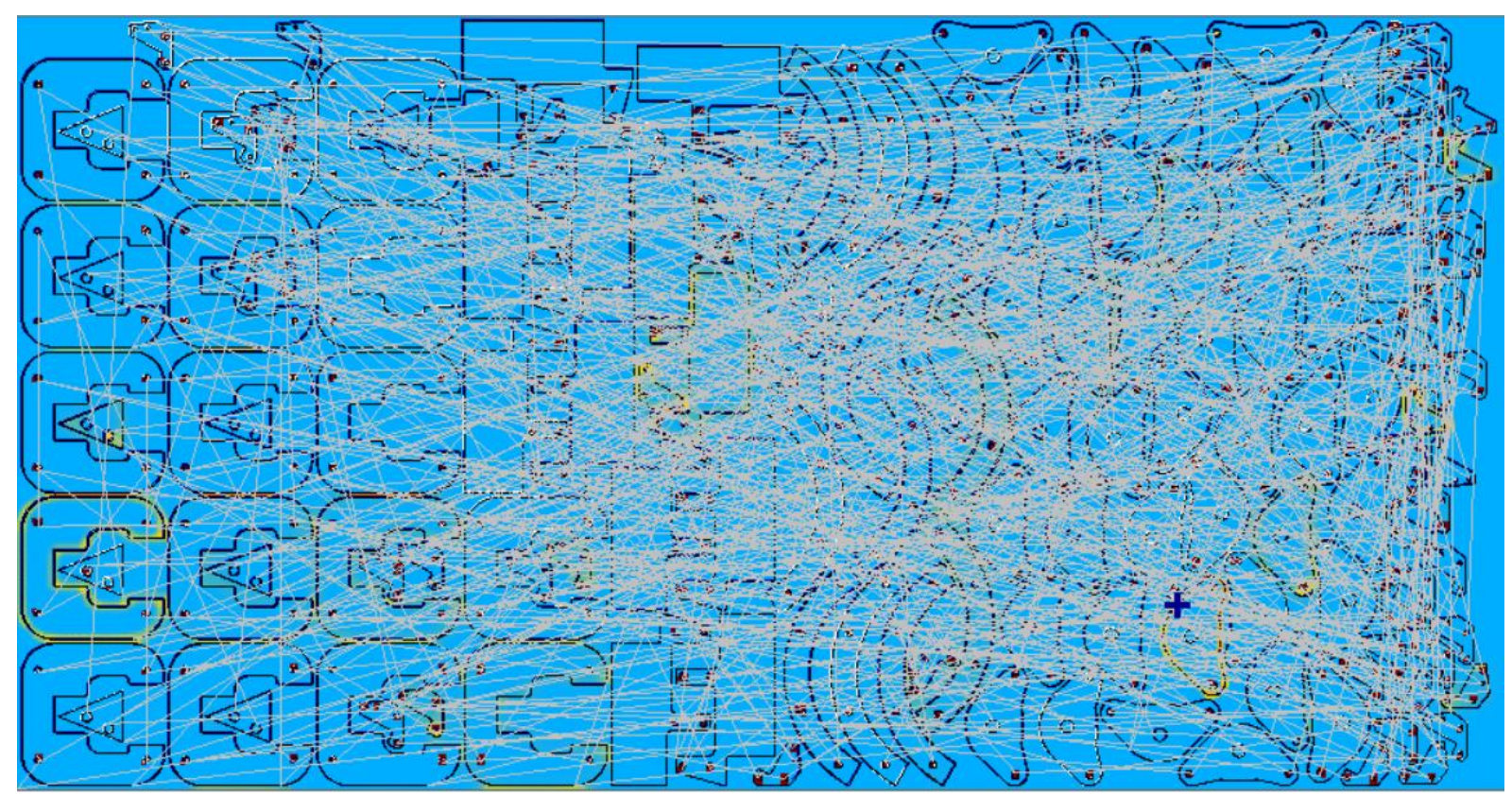

$a$

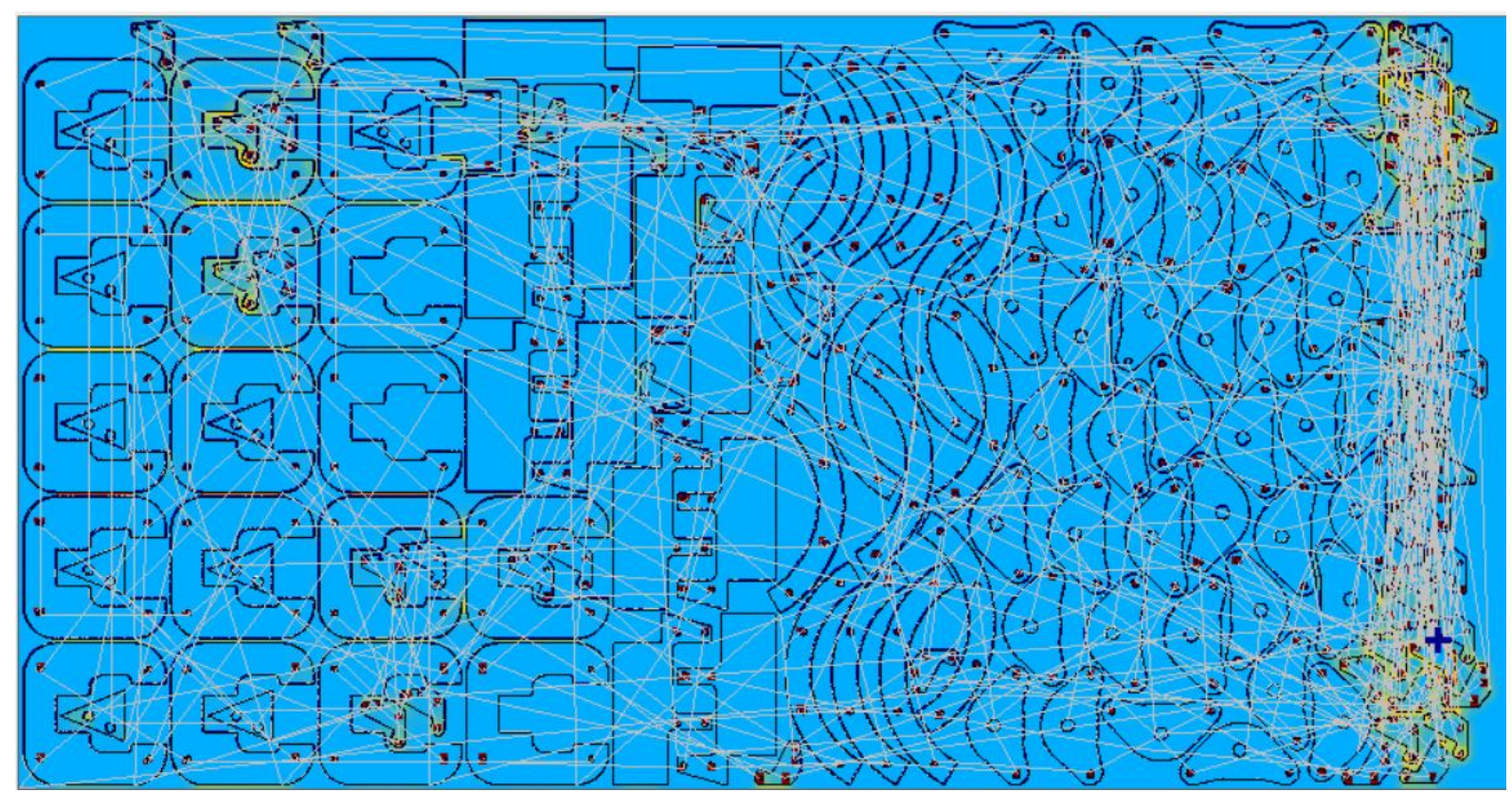

$b$

Figure 8. Examples of a cutting plan: cutting plan obtained by the "Random transitions" algorithm (a) and the "Minimize path and overheats taking into account temperature in real time" algorithm (b). 


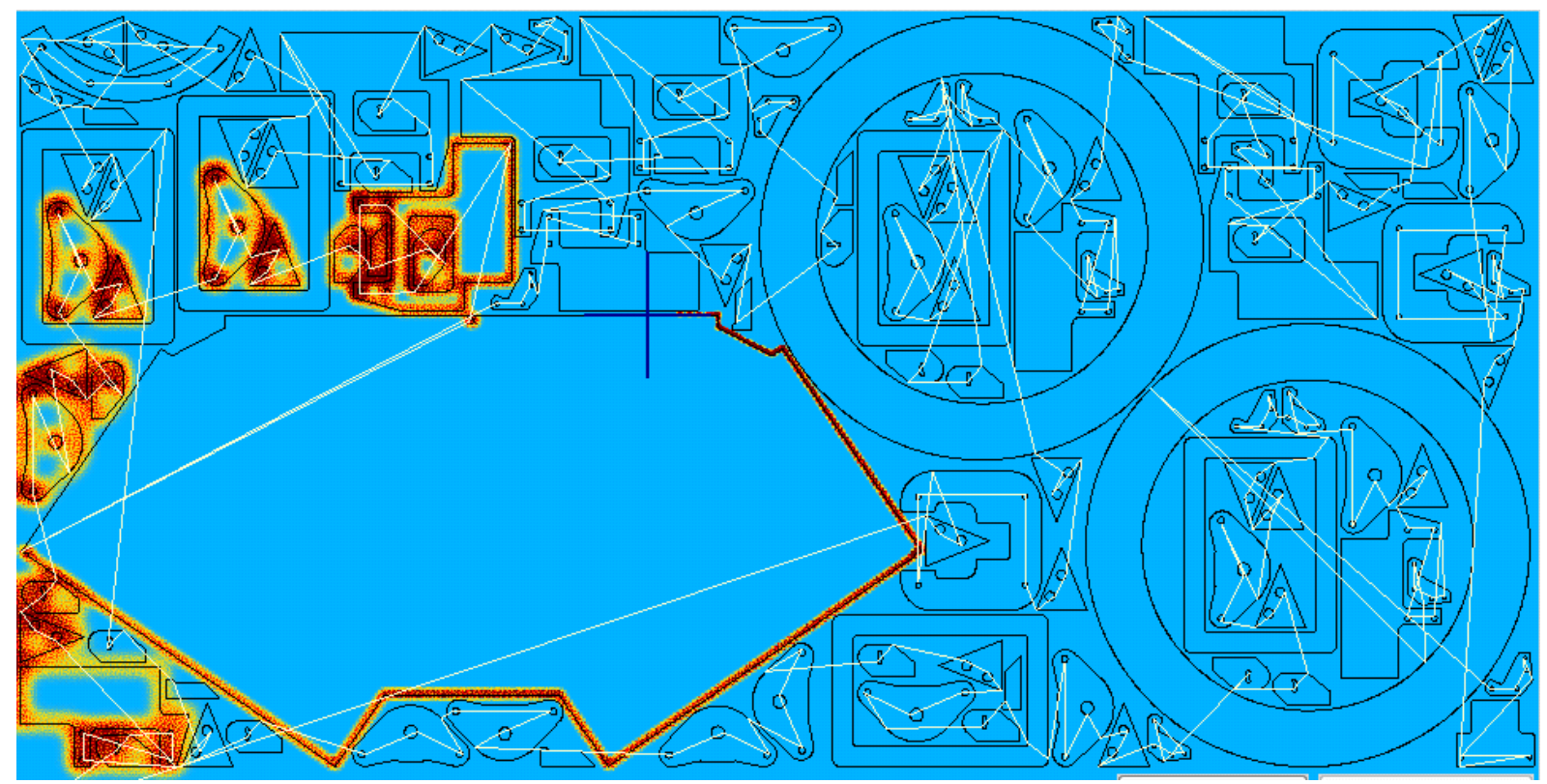

Figure 9. Cutting plan, path of the cutting tool and $T$ points of the sheet, taking into account the "Additional constraints".

\section{Conclusion}

An approach to solving the problem of designing a digital twin of the process of the thermal cutting of sheet material, taking into account thermal effects, based on a discrete-logical representation of information, including a mathematical model, methods, algorithms and software, is considered.

This makes it possible to take into account in real time the processes of heating and cooling of the cutting sheet in the laser, gas, etc. cutting sheet material into figure billets, taking into account technological limitations arising in the case of parts (billets) cutting.

\section{References}

[1] Wäscher G, Haußner H and Schumann H 2007 An improved typology of cutting and packing problems European Journal of Operational Research 183 pp 1109-1130

[2] Yang W, Zhao Y, Jie J and Wang W 2010 An effective algorithm for tool path airtime optimization during leather cutting Advanced Material Research 102-104 pp 373-377

[3] Tavaeva A, Petunin A, Ukolov S and Krotov V 2019 A cost minimizing at laser cutting of sheets parts on CNC machines Communications in Computer and Informational Science 1090 CCIS pp 422-437

[4] Tavaeva A F, Petunin A A and Polishchuk E G 2020 Methods of cutting cost minimizing in problem of tool route optimization for CNC laser machines Lecture Notes in Mechanical Engineering pp 447-455

[5] Petunin A A 2019 General model of tool path problem for the CNC sheet cutting machines IFAC - PapersOnLine 52(13) pp 2662-2667

[6] Chentsov A G, Chentsov P A, Petunin A A and Sesekin A N 2018 Model of megapolises in the tool path optimization for CNC plate cutting machines International Journal of Production Research 56(14) pp 4819-4830

[7] Petunin A A, Polishuk E G, Chentsov A G, Chentsov P A and Ukolov S S 2016 About some types of constraints in problems of routing AIP: proceedings inter. conf. $\mathbf{1 7 8 9} 060002$

[8] Levichev N, Rodrigues G C, Dewil R and Duflon J 2020 Anticipating heat accumulation in laser oxygen cutting of thick metal plates Journal of Laser Applications 32(2) 022018 
[9] Verkhoturov M, Petunin A, Verkhoturova G, Danilov K and Kurennov D 2016 The 3D object packing problem into a parallelepiped container based on discrete-logical representation IFAC-Papers OnLine 49(12) pp 1-5

[10] Verkhoturov M 2007 The two-dimensional irregular cutting stock problem: optimization allocation and path of cutting instrument Vestnik USATU 9(20) pp 106-118 (http://journal.ugatu.ac.ru/index.php/Vestnik/article/view/1412)

[11] Wilkins J 2017 How Industry 4.0 impacts globalization [Online] Available: https://www.manufacturingglobal.com/technology/how-industry-40-impacts-globalisation [Accessed 06.04.2021]

[12] Davis N 2016 What is the fourth industrial revolution? [Online] Available: https://www.weforum.org/agenda/2016/01/what-is-the-fourth-industrial-revolution [Accessed 06.04.2021]

[13] Valeev S and Kondratyeva N 2021 Process safety and big data (Cambridge, MA: Elsevier)

[14] Valeev S, Kondratyeva N and Verkhoturov M 2019 Intelligent Decision Support System of Operation Sequencing of Technological Processes Planning Proc. of 2019 International Russian Automation Conference (RusAutoCon) pp 1-6 